Pacific Journal of Mathematic 


\title{
EQUIVALENT DEFINITIONS OF POSITIVE DEFINITENESS
}

\author{
P. H. Maserick AND F. H. Szafraniec
}

\begin{abstract}
Equivalent conditions for linear functionals, on a commutative algebra $\mathbb{Q}$ with involution, to be positive are proved by elementary methods devoid of disintegration theorems. The theory is applied to present straightforward verifications of, heretofore subtle, equivalences of quadratic form and finite difference definitions of positive definiteness of functions on semigroups.
\end{abstract}

1. Introduction. Let $\mathbb{Q}$ be a commutative algebra with identity 1 and involution*. A subset $\tau$ of $\mathbb{Q}$ such that

(i) $x^{*}=x$ for all $x \in \tau$,

(ii) $1-x$ is a positive linear combination of products of members of $\tau$ for each $x \in \tau\left(1-x \in \mathrm{Alg} \operatorname{span}^{+} \tau\right.$ for each $\left.x \in \tau\right)$, and

(iii) each $x \in \mathbb{Q}$ is a linear combination of products of members of $\tau$ $(\mathbb{Q}=\operatorname{Alg} \operatorname{span} \tau)$

will be called admissible. A linear functional $f$ on $Q$ such that $f(x) \geq 0$ for all $x \in \mathrm{Alg} \operatorname{span}^{+} \tau$ where $\tau$ is admissible will be called $\tau$-positive. Following standard conventions, $f$ is called positive if $f\left(x x^{*}\right) \geq 0$ for all $x \in \mathbb{Q}$. If $f$ is positive then we set $|x|_{f}^{2}=\sup _{y}\left(f\left(x x^{*} y y^{*}\right) / f\left(y y^{*}\right)\right)(0 / 0=$ 0 ) and say $f$ is bounded whenever $|x|_{f}<\infty$ for each $x \in \mathbb{Q}$. It is shown in Maserick [8, Th. 1.1] that every $\tau$-positive linear functional $f$ admits a disintegration

$$
f(x)=\int_{\Gamma} \rho(x) d \mu_{f}(\rho)
$$

where $\mu_{f}$ is a non-negative regular Borel measure on a compact (weak*-topology) subset $\Gamma$ of the $\tau$-positive multiplicative linear functionals. From this it follows that every $\tau$-positive linear functional is bounded and positive. The point of this paper is to give an elementary and almost completely algebraic proof of this latter fact, which does not appeal to the disintegration theorem cited above. By way of motivation, consider the following classical examples from analysis.

EXAMPLE 1.1. The "little" moment problem. Let $\mathcal{Q}$ be the real algebra of polynomials in one variable with $p^{*}=p$ for all $p \in \mathbb{Q}$ and let $\tau$ consist of the two polynomials $p_{1}(t)=t$ and $p_{2}(t)=1-t$. The linear functionals 
$L$ on $Q$ can be identified with the sequences $a=\left\{a_{n}\right\}_{n}$ via the moment relation $L\left(\sum_{n} \alpha_{n} t^{n}\right)=\Sigma_{n} \alpha_{n} a_{n}$. Since Alg $\operatorname{span}^{+} \tau$ is the positive linear span of the polynomials of the form $t^{m}(1-t)^{n}$, a positive linear functional identifies with a sequence if and only if all finite differences of the sequences are non-negative, i.e. $\left\{a_{n}\right\}_{n}$ is a completely monotonic sequence cf. [12, p. 108]; the $\tau$-positive multiplicative functionals being identified with the sequences $\left\{t^{n}\right\}_{n}$ for $0 \leq t \leq 1\left(0^{0}=1\right) \mathrm{cf}$. [8, p. 147]. Under this identification, (1.0.1) reduces to

$$
a_{n}=\int_{0}^{1} t^{n} d \mu_{a}(t)
$$

the "little" moment problem of Hausdorff. On the other hand, a sequence becomes positive under this identification when and only when the moments of the squares of polynomials are non-negative, i.e. when all quadratic forms of the type $\Sigma_{i, j} c_{i} c_{j} a_{i+j}\left(c_{i} \in \mathbf{R}\right)$ are non-negative. It is well known and follows from (1.0.2) that a sequence $\left\{a_{n}\right\}_{n=0}$ is completely monotonic if and only if both $\left\{a_{n}\right\}_{n=0}$ and $\left\{a_{n}-a_{n+1}\right\}_{n=0}$ are positive definite. In [1, p. 325], Atzmon asks for a direct proof (i.e. a proof that does not use (1.0.2)) of this fact. The theory of reproducing kernels can be used to supply the direct proof that both positive definite conditions together imply complete monotonicity, while the main theorem of this paper yields the requested proof of the converse. This is done in Application (4.2). More generality is obtained by replacing $\mathbb{Q}$ above by the algebra of shift operators on a commutative semigroup $S$. Atzmon's question is then answered by specializing $S$ to the additive semigroup of non-negative integers.

EXAMPLE 1.2. The trigonometric moment problem of Herglotz. Let $Q$ be the complex algebra of trigonometric polynomials $\Sigma_{n} \alpha_{n} e^{i n \theta}$ and let

$$
\tau=\left\{\frac{1}{2}\left(1+(\sigma / 2) e^{i \theta}+(\bar{\sigma} / 2) e^{-i \theta}\right) \mid \sigma^{4}=1\right\} .
$$

Similar to the previous example, the linear functionals $L$ can be identified with functions $f$ on the group $I$ of integers via the moment relation $L\left(\Sigma_{n} \alpha_{n} e^{i n \theta}\right)=\Sigma_{n} \alpha_{n} f(n)$; the positive multiplicative functionals being of the form $n \rightarrow e^{i n \theta}(n \in I, \theta \in[0,2 \pi))$. A characterization, in terms of finite differences, of those functions $f$ on $I$ which correspond to the $\tau$-positive linear functionals on $\mathcal{Q}$ is given in [7, p. 141] and the integral representation (1.0.1) reduces to the form of the trigonometric moment problem, namely

$$
f(n)=\int_{[0,2 \pi)} e^{i n \theta} d \mu_{f}(\theta)
$$


But Hergoltz [5, p. 773] has characterized those functions $f$ satisfying (1.0.3) as the positive definite functions on $I$. Thus a function $f$ on $I$ is positive definite if and only if it satisfies the appropriate finite difference condition. In Application (4.3) we give an alternate proof of this equivalence, in considerably more generality, which does not depend on (1.0.3).

EXAMPLE 1.3. The non-negative, non-decreasing real functions on $(0,1]$. Let $\tau$ be the characteristic functions $\left\{1_{(a, b]} \mid 0<a<b \leq 1\right\}$ and $\mathbb{Q}$ the real algebra, with trivial involution, spanned by $\tau$. The equation $L\left(1_{(0, a]}\right)=f(a)$ identifies the linear functionals $L$ on $Q$ with the real functions $f$ on $(0,1]$; the positive linear functionals being identified with the non-negative, non-decreasing functions. The main theorem (Th. 3.1) then implies that a function $f$ on $(0,1]$ is non-negative and non-decreasing if and only if the matrix $\left(f\left(\min \left[x_{i}, x_{j}\right]\right)\right)_{n \times n}$ is positive definite for every $n$.

2. $\tau$-positiveness of bounded positive functionals. For each $x \in \mathbb{Q}$, define the shift operator $E_{x}$ on the linear functionals $f$ on $\mathbb{Q}$ by $E_{x} f(y)=$ $f(x y)(y \in \mathbb{Q})$. Let $f$ be a fixed bounded positive linear functional and set $H_{f}=\left\{E_{x} f \mid x \in \mathbb{Q}\right\}$. Parts (i) and (iii) of $\S 1$ imply $f\left(x^{*}\right)=\bar{f}(x)$. Thus $H_{f}$ can be made into a pre-Hilbert space in a manner similar to the GelfandSegal-Naimark (GNS) construction $\left[9\right.$, p. 215] by defining $\left\langle E_{x} f, E_{y} f\right\rangle=$ $f\left(x y^{*}\right)$. The Cauchy-Schwarz inequality shows

$$
\left|E_{x} f(y)\right|^{2}=\left|\left\langle E_{x} f, E_{y^{*}} f\right\rangle\right|^{2} \leq\left\langle E_{x} f, E_{x} f\right\rangle \cdot\left\langle E_{y^{*}} f, E_{y^{*}} f\right\rangle
$$

or

$$
\left|E_{x} f(y)\right| \leq\left\|E_{y^{*}} f\right\| \cdot\left\|E_{x} f\right\| .
$$

Thus if $\left\|E_{x} f\right\|=0$ then $E_{x} f=0$, so $\langle\cdot, \cdot\rangle$ is a non-degenerate inner product. Substituting $\left(E_{x_{n}}-E_{x_{m}}\right)$ for $E_{x}$ in (2.0.1) shows that every Cauchy sequence in $H_{f}$ is weak*-Cauchy and thus the completion $\bar{H}_{f}$ is a subset of the linear functionals on $\mathcal{Q}$. For each $x \in \mathbb{Q}, E_{x}$ is then a bounded linear operator on the pre-Hilbert space $H_{f}$ (i.e. $E_{x} \in B\left(H_{f}\right)$ ) with operator norm

$$
\left\|E_{x}\right\|=|x|_{f}
$$

Since Cauchy sequences in $H_{f}$ are also weak*-Cauchy, it follows that the extension of $E_{x}$ to the completion $\bar{H}_{f}$ of $H_{f}$ is again the translation operator $E_{x}$. Thus the map $x \rightarrow E_{x}$ of $Q$ onto $B\left(\bar{H}_{f}\right)$ is a (*-preserving) representation of $\mathcal{Q}$ such that $E_{1}$ is the identity operator $I$. The following proposition summarizes the pertinent facts. 


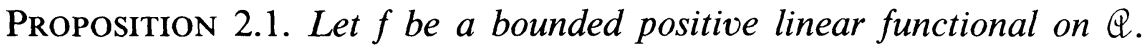
Then:

(i) The operator $E_{x} \in B\left(\bar{H}_{f}\right)$ is positive if and only if $E_{x} f$ is a positive linear functional on $Q$.

(ii) If $\tau$ is admissible and $E_{x} f$ is positive for each $x \in \tau$ then $f$ is $\tau$-positive.

(iii) If $\alpha_{x}$ is any positive number such that $\alpha_{x} \geq|x|_{f}$ then

$$
\tau=\left\{\frac{1}{2}\left(I+\left(\sigma / 2 \alpha_{x}\right) x+\left(\bar{\sigma} / 2 \alpha_{x}\right) x^{*}\right) \mid x \in \mathbb{Q}, \sigma^{4}=1\right\}
$$

is admissible and $f$ is $\tau$-positive.

Proof. Assertion (i) is obvious from the foregoing remarks. For (ii), let $x_{1}, \ldots, x_{k} \in \tau$. If $f$ satisfies the hypothesis of (ii) then $E_{x_{j}}$ is a positive operator on $\bar{H}_{f}$ for each $j=1, \ldots, k$ by (i). Since products of commuting bounded positive operators are again positive (cf. [6, p. 280] for a proof of this latter fact which does not depend on the spectral theorem), one has $f\left(\Pi_{j} x_{j}\right)=\left\langle E_{\Pi_{j} x_{j}} f, f\right\rangle \geq 0$ or $f$ is $\tau$-positive. Admissibility of $\tau$ in assertion (iii) is easily verified. But if $A$ is any non-zero bounded operator on a Hilbert space and $\alpha \geq\|A\|$ the $I+(\sigma / 2 \alpha) A+(\bar{\sigma} / 2 \alpha) A^{*}$ is positive. Thus (2.0.2) implies the operator $I+\left(\sigma / 2 \alpha_{x}\right) E_{x}+\left(\bar{\sigma} / 2 \alpha_{x}\right) E_{x}^{*}$ is positive on $\bar{H}_{f}$ so that (iii) follows from (ii).

3. Positiveness of bounded positive functionals. We now give the elementary proof promised in the introduction. Namely,

THEOREM 3.1. If $f$ is $\tau$-positive for an admissible $\tau$, then $f$ is positive and bounded, and $E_{x} f$ is positive for each $x \in \tau$.

Proof. (i) The central idea of the proof revolves around the theory of Bernstein polynomials. If $p\left(t_{1}, t_{2}\right)$ is any complex polynomial in two variables, $x_{1}, x_{2} \in \mathbb{Q}$ and $n$ is any positive integer, we define

$$
\begin{aligned}
& B_{n}\left(p\left(x_{1}, x_{2}\right)\right) \\
& \quad=\sum_{i, j} p\left(\frac{i}{n}, \frac{j}{n}\right)\left(\begin{array}{c}
n \\
i
\end{array}\right)\left(\begin{array}{c}
n \\
j
\end{array}\right) x_{1}^{i} x_{2}^{j}\left(1-x_{1}\right)^{n-t}\left(1-x_{2}\right)^{n-j} .
\end{aligned}
$$

Letting $p\left(x_{1}, x_{2}\right)=\left(x_{1}-x_{2}\right)^{2}$, we get

$$
\begin{aligned}
B_{n}\left(x_{1}-x_{2}\right)^{2} & =B_{n}\left(x_{1}^{2}-2 x_{1} x_{2}+x_{2}^{2}\right) \\
= & \left(B_{n}\left(x_{1}^{2}\right)\right)\left(B_{n}(1)\right)-2\left(B_{n}\left(x_{1}\right)\right)\left(B_{n}\left(x_{2}\right)\right)+\left(B_{n}(1)\right)\left(B_{n}\left(x_{2}^{2}\right)\right) \\
= & B_{n}\left(x_{1}^{2}\right)-2 x_{1} x_{2}+B_{n}\left(x_{2}^{2}\right) .
\end{aligned}
$$


But, $B_{n}\left(x^{2}\right)=x / n+((n-1) / n) x^{2}($ cf. Davis [4, p. 109]) so that

$$
\begin{aligned}
B_{n}\left(x_{1}-x_{2}\right)^{2}= & {\left[\frac{x_{1}}{n}+\left(\frac{n-1}{n}\right) x_{1}^{2}\right]-\left[2 x_{1} x_{2}\right] } \\
& +\left[\frac{x_{2}}{n}+\left(\frac{n-1}{n}\right) x_{2}^{2}\right] .
\end{aligned}
$$

If we impose any locally convex topology on $Q$, such as the weakest in which all linear functionals are continuous, then we have

$$
\begin{aligned}
\lim _{n} E_{x} f\left(B_{n}\left(x_{1}-x_{2}\right)^{2}\right) & =\lim _{n} f\left(x B_{n}\left(x_{1}-x_{2}\right)^{2}\right) \\
& =f\left(x\left(x_{1}-x_{2}\right)^{2}\right)=E_{x} f\left(\left(x_{1}-x_{2}\right)^{2}\right) .
\end{aligned}
$$

But if $x, x_{1}, x_{2} \in \operatorname{Alg} \operatorname{span}^{+} \tau$ then [8, Prop. 2.1.1] implies both $1-x_{1} / M$, $1-x_{2} / M \in \mathrm{Alg} \operatorname{span}^{+} \tau$ for sufficiently large $M>0$. Since the coefficients of $B_{n}\left(x_{1} / M-x_{2} / M\right)^{2}$ are non-negative, linearity of $f$ implies $E_{x} f\left(\left(x_{1} / M-x_{2} / M\right)^{2}\right) \geq 0$ so that

$$
E_{x} f\left(\left(x_{1}-x_{2}\right)^{2}\right) \geq 0
$$

Let $y \in \mathbb{Q}$. By condition (iii) of the definition of $\tau, y=\left(x_{1}-x_{2}\right)+$ $\left(x_{3}-x_{4}\right) i$ where $x_{j} \in \operatorname{Alg} \operatorname{span}^{+} \tau$ for each $j=1,2,3$ or 4 . Thus $y y^{*}=$ $\left(x_{1}-x_{2}\right)^{2}+\left(x_{3}-x_{4}\right)^{2}$ so that (3.1.3) and linearity of $E_{x} f$ imply

$$
E_{x} f\left(y y^{*}\right) \geq 0 \text {. }
$$

Hence $E_{x} f$ is positive for each $x \in \operatorname{Alg} \operatorname{span}^{+} \tau$. But if $x \in \tau$ then $1-x \in \mathrm{Alg} \operatorname{span}^{+} \tau$, and since $E_{(1-x)}=I-E_{x}$ we have $f=E_{x} f+$ $\left(I-E_{x}\right) f$, so that $f$ is positive. This establishes the first and last assertions.

(ii) To see that $f$ is bounded, let $z$ be an arbitrary member of $\tau$ and replace $x$ in $(3.1 .3)$ by $1-z^{2}$. Clearly $1-z^{2}=(1-z)(1+z) \in$ $\mathrm{Alg} \operatorname{span}^{+} \tau$ so that (3.1.4) and Proposition 2.1 (i) imply $E_{1-z^{2}}=I-E_{z^{2}}$ is a positive operator on $H_{f}$. It follows that $E_{z}$ is bounded in norm by 1 for all $z \in \tau$. But since $\tau$ generates $Q, E_{x}$ is bounded for all $x \in \mathbb{Q}$. Therefore $f$ is bounded.

4. Applications to semigroups. Let $S$ be a commutative semigroup with identity $e$ and involution*. To apply the theory presented in the foregoing sections, define the shift operator $E_{s}$ on the space $\mathscr{F}(S)$ of complex-valued functions $\varphi$ on $S$ by $\left(E_{s} \varphi\right)(t)=\varphi(s t)(s, t \in S)$ and set $\mathbb{Q}=\operatorname{span}\left\{E_{s} \mid s \in S\right\}$. Since $E_{s t}=E_{s} E_{t}, \mathbb{Q}$ is an algebra with identity $I=E_{e}$ and involution $\left(\Sigma_{i} c_{i} E_{s_{i}}\right)^{*}=\Sigma_{i} \bar{c}_{i} E_{s_{i}^{*}}$. The linear functionals $f$ on $\mathbb{Q}$ 
can be biuniquely identified with the functions $\varphi \in \mathscr{F}(S)$ by $f\left(\sum c_{i} E_{s_{i}}\right)=$ $\sum c_{\imath} \varphi\left(s_{i}\right)$; the positive functionals being identified with those $\varphi$ satisfying

$$
\sum_{i, j} c_{l} \bar{c}_{j} \varphi\left(s_{i} s_{j}^{*}\right) \geq 0
$$

for all finite subsets $\left\{c_{i}\right\}_{i} \subset \mathbf{C}$ and $\left\{s_{i}\right\}_{l} \subset S$. Consistent with standard terminology when $S$ is a group, we call those functions which satisfy (4.0.1) for an arbitrary semigroup $S$, positive definite. In this generality, bounded positive definite functions were first introduced and studied in [5] under the name "*-definite". The GNS-construction used in $\$ 2$ carries over to the set $H_{\varphi}=\{T \varphi \mid T \in \mathbb{Q}\}$ giving a pre-Hilbert space with $\langle T \varphi, R \varphi\rangle=T R^{*} \varphi(e)$. Moreover the operator $E_{T}$ on $H_{f}$ corresponds to the operator $T$ on $H_{\varphi}$ defined by $T\left(T_{1} \varphi\right)=T T_{1}(\varphi)$. In particular $E_{s}$ is an operator on $H_{\varphi}$ which corresponds to the operator $E_{\left(E_{s}\right)}$ on $H_{f}$. This construction on $\mathbb{Q}$ reduces to the theory of reproducing kernels on $S \times S$ relative to the positive definite kernel $k(s, t)=\varphi\left(s t^{*}\right)$. The following preliminary proposition is needed.

Proposition 4.1. If $\varphi \in \mathcal{F}(S)$ is positive definite in the sense of (4.0.1) and bounded in the usual sense, then $E_{s}$, as an operator on $H_{\varphi}$, is bounded in norm by 1.

Proof. (i) If $\psi$ is any positive definite function on $S$ then the CauchySchwarz inequality yields

$$
\left|\psi\left(s t^{*}\right)\right|^{2}=\left|\left\langle E_{s} \psi, E_{t} \psi\right\rangle\right|^{2} \leq\left\|E_{s} \psi\right\|^{2} \cdot\left\|E_{t} \psi\right\|^{2}=\psi\left(s s^{*}\right) \psi\left(t t^{*}\right)
$$

Thus

$$
\left|\psi\left(s t^{*}\right)\right|^{2} \leq \psi\left(s s^{*}\right) \psi\left(t t^{*}\right)
$$

so that setting $t=e$ gives

$$
|\psi(s)|^{2} \leq \psi(e) \psi\left(s s^{*}\right)
$$

(ii) If $\psi$ is bounded and positive definite then $|\psi(s)| \leq \psi(e)$ for all $s \in S$. Indeed, we may assume $\psi(e)=1$ without loss of generality. For $s \in S$ and $a=s s^{*}$, we find by $p$ successive applications of (4.1.2) that $|\psi(s)|^{2^{p}} \leq \psi\left(a^{2^{p-1}}\right)$. But if $\psi$ is bounded by $K$, then $|\psi(s)| \leq K^{2^{-p}} \rightarrow 1=$ $\psi(e)$ (cf. Szafraniec [10, 11] or Berg-Maserick [2]). 
(iii) Let $T \in \mathbb{Q}$. Then $T T^{*} \varphi$ is positive definite since $E_{T T^{*}} f$ is a positive linear functional. Thus

$$
\begin{aligned}
T T^{*} \varphi\left(s s^{*}\right) & =\left\langle E_{s s^{*}} \varphi, T T^{*} \varphi\right\rangle \leq\left\|E_{s s^{*}} \varphi\right\| \cdot\left\|T T^{*} \varphi\right\| \\
& =\sqrt{\varphi\left(\left(s s^{*}\right)^{2}\right)}\left\|T T^{*} \varphi\right\| \leq \sqrt{\varphi(e)}\left\|T T^{*} \varphi\right\| .
\end{aligned}
$$

Setting $\psi=T T^{*} \varphi$ in (4.1.2) shows $T T^{*} \varphi$ is bounded, from which (ii) implies $\left|T T^{*} \varphi(s)\right| \leq T T^{*} \varphi(e)$. Thus

$$
\left|\left\langle E_{s} T \varphi, T \varphi\right\rangle\right|=\left|T T^{*} \varphi(s)\right| \leq T T^{*} \varphi(e)=\langle T \varphi, T \varphi\rangle
$$

so $\left\|E_{s}\right\| \leq 1$.

The applications depend on appropriate selections of $\tau$ which in turn may depend on the choice of $S$. In the interest of brevity we have selected two representative examples below for illustration. However, virtually all of the applications appearing in Berg-Maserick [2, \$4] could be recast in this more elementary point of view, in lieu of the disintegration theorems used therein. Throughout $G$ will denote a generator set for $S$ in the sense that every $s \in S$ is a finite product of the form $\Pi_{i} s_{i}$, where either $s_{i} \in G$ or $s_{i}^{*} \in G$ for each $i$.

(A) Completely monotonic functions. We assume $s=s^{*}$ for all $s \in S$ and recall that finite differences of a function $\varphi \in \mathscr{F}(S)$ are defined inductively by

$$
\begin{aligned}
& \Delta_{k+1} \varphi\left(s_{0} ; s_{1}, \ldots, s_{k+1}\right) \\
& =\Delta_{k} \varphi\left(s_{0} ; s_{1}, \ldots, s_{k}\right)-\Delta_{k} \varphi\left(s_{0} s_{k+1} ; s_{1}, \ldots, s_{k}\right) \quad(k=1,2, \ldots),
\end{aligned}
$$

where $s_{0}, s_{1}, \ldots, s_{k+1} \in S$. A non-negative $\varphi \in \mathscr{F}(S)$ is said to be completely monotonic if all of the differences (4.1.3) are non-negative. It is easy to see that these differences can be redefined in terms of the shift operator by

$$
\Delta_{k} \varphi\left(s_{0} ; s_{1}, \ldots, s_{k}\right)=E_{s_{0}} \prod_{i}\left(I-E_{s_{\imath}}\right) \varphi(e) .
$$

It follows that $\varphi$ is completely monotonic if and only if the linear functional $f$ corresponding to $\varphi$ is $\tau$-positive with respect to $\tau=\left\{E_{s}\right.$, $\left.I-E_{s} \mid s \in S\right\}$. However, we can reduce the size of $\tau$ to

$$
\tau_{G}=\left\{E_{s}, I-E_{s} \mid s \in G\right\}
$$


without adding more $\tau$-positive functionals because it follows (algebraically, cf. Maserick [8, Prop. 2.1.1]) that $\left\{E_{s}, I-E_{s}\right\} \subset \mathrm{Alg} \mathrm{Span}^{+} \tau_{G}$ for each $s \in S$. Hence $\operatorname{Alg} \operatorname{Span}^{+} \tau_{G}=\operatorname{Alg} \operatorname{Span}^{+} \tau$. Thus $\varphi \in \mathscr{F}(S)$ is completely monotonic if and only if $\Pi_{l=1}^{k}\left(I-E_{s_{t}}\right)\left(s_{0}\right) \geq 0$ for all $s_{0}, s_{1}, \ldots, s_{k} \in G$ where $G$ is any generator set whatsoever.

The proof of the equivalence of (i) and (ii) below answers the question of Atzmon, mentioned in the introduction, by taking $S$ to be the additive semigroup of non-negative integers.

Application 4.2. Let $\varphi \in \mathscr{F}(S)$. The following are equivalent.

(i) $\varphi$ is completely monotonic,

(ii) $E_{s} \varphi$ and $\left(I-E_{s}\right) \varphi$ are positive definite for each $s \in G$, and

(iii) $\varphi$ is bounded and $E_{s} \varphi$ is positive definite for each $s \in G \cup\{e\}$.

Proof. The implication (i) implies (ii) follows from the foregoing remarks and Theorem 3.1. Condition (ii) implies boundedness of $\varphi$ on $G$. This, in turn, implies boundedness of $\varphi$ on $S$ since $I-E_{s}$ is in the positive linear span of $\left\{I-E_{t} \mid t \in G\right\}$ for all $s \in S$ as mentioned above. Thus (ii) implies (iii). Assuming (iii), Proposition (4.1) implies $\left\|E_{s}\right\| \leq 1$ for all $s \in G$ and since the operator $E_{s}$ is positive by (i) of Proposition 2.1, it follows that $I-E_{s}$ is also positive. Commutativity implies all finite products from the set $\left\{E_{s}, I-E_{s} \mid s \in G\right\}$ are also positive operators so that

$$
\prod_{i}\left(I-E_{s_{t}}\right) \varphi\left(s_{0}\right)=\left\langle E_{s_{0}} \prod_{i}\left(I-E_{s_{t}}\right) \varphi, \varphi\right\rangle \geq 0 \quad \text { for } s_{0}, s_{1}, \ldots \in G
$$

and (i) follows from (iii).

REMARK. The proof of implications (i) $\Rightarrow$ (ii) $\Rightarrow$ (iii), without appealing to disintegration theory, is new to the best of our knowledge. We do not claim novelty here for the proof that (iii) implies (i); cf. [5, Cor. 4.4].

(B) Trigonometric moment problem. We now allow a nontrivial involution on $S$.

Application 4.3. Let $\varphi$ be a bounded function on $S, G$ a generator set of $S$ and $\tau_{G}=\left\{\frac{1}{2}\left(I+(\sigma / 2) E_{s}+(\bar{\sigma} / 2) E_{s^{*}}\right) \mid \sigma^{4}=1, s \in G\right\}$. The following are equivalent:

(i) $\varphi$ is $\tau_{G}$-positive.

(ii) $\varphi$ is positive definite. 
Proof. Since $\tau_{G}$ is admissible, Theorem 3.1 shows (i) implies (ii). Assuming (ii), Proposition (4.1) implies $\left\|E_{s}\right\| \leq 1$ for all $s \in S$, since $\varphi$ is bounded. Standard operator theory implies $\left(I+(\sigma / 2) E_{s}+(\bar{\sigma} / 2) E_{s^{*}}\right)$ is positive for all 4 th roots of unity $\sigma$ and $s \in S$. Using commutativity as before, one finds

$$
\prod_{j=1}^{k} T_{j} \varphi(e)=\left\langle\prod_{j=1}^{k} T_{j} \varphi, \varphi\right\rangle \geq 0
$$

whenever $T_{j} \in \tau_{G}$ so that (i) follows from (ii).

REMARKs. (a) It now follows that the definition of $\tau_{C}$-positivity is independent of the choice of $G$ for bounded $\varphi$. This is not the case, however, if $\varphi$ is unbounded [7, p. 142].

(b) If a positive definite function $\varphi$ is bounded on $\left\{s s^{*} \mid s \in S\right\}$ then (4.1.2) shows $\varphi$ to be bounded on $S$ itself. If, for example, $\left(s s^{*}\right)$ is idempotent for each $s \in S$, then a second application of (4.1.2) shows that every positive definite $\varphi$ is bounded. Since without any boundedness restriction $\tau_{G}$-positivity implies positive definiteness, boundedness can be waived for this rather general case. This idempotency occurs when $s$ is a group with inversion as involution as in Example (1.2), or when the operation on $S$ itself is idempotent such as Example (1.3) $(s t=\min [s, t])$ and $S$ has the trivial involution or more generally when $S$ is an inverse semigroup with $s^{*}=s^{-1}$ (cf. Clifford and Preston [3]).

(c) If $\varphi$ is $\tau_{s}$-positive then $\varphi$ is bounded on $\left\{s s^{*} \mid s \in S\right\}$ so that boundedness is not needed to prove that (i) implies (ii) but is needed to show the converse [7, p. 142].

\section{REFERENCES}

[1] A. Atzmon, A moment problem for positive measures on the unit disc., Pacific J. Math., 59 (1975), 317-325.

[2] C. Berg and P. H. Maserick, Exponentially bounded positive definite functions, submitted.

[3] A. H. Clifford, G. B. Preston, The Algebraic Theory of Semigroups. Vol. I, Math. Surveys, no. 7, Amer. Math. Soc., Providence, R.I., 1961.

[4] Philip J. Davis, Interpolation and Approximation, Blaisdell Publishing Co., New York, 1963.

[5] R. J. Lindahl and P. H. Maserick, Positive-definite functions on involution semigroups, Duke Math. J., 38 (1971) 771-782.

[6] L. A. Lusternik and V. J. Sobolev, Elements of Functional Analysis. New York, 1968.

[7] P. H. Maserick, $B V$-functions, positive-definite functions and moment problems, Trans. Amer. Math. Soc., 214 (1975), 137-152.

[8] _ Moments of measures on convex bodies., Pacific J. Math., 68 (1977), 135-152. 
[9] Charles E. Rickart, General Theory of Banach Algebras, Van Nostrand Co., Inc. Princeton, N.J., 1960.

[10] F. H. Szafraniec, On the boundedness condition involved in dilation theory. Bull. Acad. Polon. Sci., Sér. Sci. Math., 25 (1976), 877-881.

[11] _ Dilations on involution semigroups, Proc. Amer. Math. Soc., 66 (1977), 30-32.

[12] D. V. Widder, The Laplace Transform, Princeton Math. Series, vol. 6, Princeton Univ. Press., Princeton, N.J. 1941.

Received December 22, 1981 and in revised form August 30, 1982.

The Pennsylvania State University

UNIVERSITY PARK, PA 16802 


\title{
PACIFIC JOURNAL OF MATHEMATICS EDITORS
}

\author{
DONALD BABBITT (Managing Editor) \\ University of California \\ Los Angeles, CA 90024 \\ Hugo Rossi \\ University of Utah \\ Salt Lake City, UT 84112 \\ C. C. MOORE and Arthur Ogus \\ University of California \\ Berkeley, CA 94720
}

\author{
J. Dugundir \\ Department of Mathematics \\ University of Southern California \\ Los Angeles, CA 90089-1113
}

R. FINN and H. SAMELSON

Stanford University

Stanford, CA 94305

ASSOCIATE EDITORS
R. ARENS
E. F. BECKENBACH
B. H. NeumanN
F. WOLF
K. YosHIDA (1906-1982)

\section{SUPPORTING INSTITUTIONS}

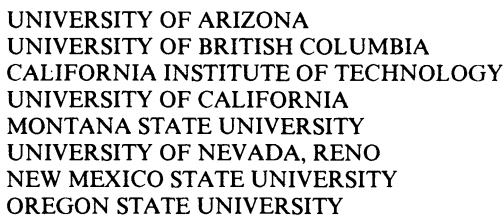

\author{
UNIVERSITY OF OREGON \\ UNIVERSITY OF SOUTHERN CALIFORNIA \\ STANFORD UNIVERSITY \\ UNIVERSITY OF HAWAII \\ UNIVERSITY OF TOKYO \\ UNIVERSITY OF UTAH \\ WASHINGTON STATE UNIVERSITY \\ UNIVERSITY OF WASHINGTON
}

The Supporting Institutions listed above contribute to the cost of publication of this Journal, but they are not owners or publishers and have no responsibility for its content or policies.

Mathematical papers intended for publication in the Pacific Journal of Mathematics should be in typed form or offset-reproduced (not dittoed), double spaced with large margins. Please do not use built up fractions in the text of the manuscript. However, you may use them in the displayed equations. Underline Greek letters in red, German in green, and script in blue. The first paragraph must be capable of being used separately as a synopsis of the entire paper. In particular it should contain no bibliographic references. Please propose a heading for the odd numbered pages of less than 35 characters. Manuscripts, in triplicate, may be sent to any one of the editors. Please classify according to the scheme of Math. Reviews, Index to Vol. 39. Supply name and address of author to whom proofs should be sent. All other communications should be addressed to the managing editor, or Elaine Barth, University of California, Los Angeles, California 90024.

There are page-charges associated with articles appearing in the Pacific Journal of Mathematics. These charges are expected to be paid by the author's University, Government Agency or Company. If the author or authors do not have access to such Institutional support these charges are waived. Single authors will receive 50 free reprints; joint authors will receive a total of 100 free reprints. Additional copies may be obtained at cost in multiples of 50 .

The Pacific Journal of Mathematics is issued monthly as of January 1966. Regular subscription rate: \$132.00 a year (6 Vol., 12 issues). Special rate: $\$ 66.00$ a year to individual members of supporting institutions.

Subscriptions, orders for numbers issued in the last three calendar years, and changes of address should be sent to Pacific Journal of Mathematics, P.O. Box 969, Carmel Valley, CA 93924, U.S.A. Old back numbers obtainable from Kraus Periodicals Co., Route 100, Millwood, NY 10546.

The Pacific Journal of Mathematics ISSN 0030-8730 is published monthly by the Pacific Journal of Mathematics at P.O. Box 969, Carmel Valley, CA 93924. Application to mail at Second-class postage rates is pending at Carmel Valley, California, and additional mailing offices. Postmaster: Send address changes to Pacific Journal of Mathematics, P. O. Box 969, Carmel Valley, CA 93924.

PUBLISHED BY PACIFIC JOURNAL OF MATHEMATICS, A NON-PROFIT CORPORATION

Copyright $(1984$ by Pacific Journal of Mathematics 


\section{Pacific Journal of Mathematics}

Vol. 110, No. $2 \quad$ October, 1984

Robert A. Bekes, The range of convolution operators $\ldots \ldots \ldots \ldots \ldots 257$

Dennis K. Burke and Sheldon Davis, Subsets of ${ }^{\omega} \omega$ and generalized metric

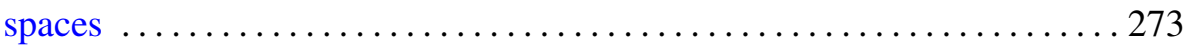

Giovanni Emmanuele, A remark on a paper: "Common fixed points of nonexpansive mappings by iteration" $\ldots \ldots \ldots \ldots \ldots \ldots \ldots \ldots \ldots 283$

I. Erdélyi and Sheng-Wang Wang, On strongly decomposable operators . . . 287

Gerhard Gierz, Injective Banach lattices with strong order units . . . . . . . 297

Maurizio Letizia, Quotients by complex conjugation of nonsingular quadrics and cubics in $\mathbf{P}_{\mathbf{C}}^{3}$ defined over $\mathbf{R} \ldots \ldots \ldots \ldots \ldots \ldots \ldots \ldots \ldots \ldots$

P. H. Maserick and Franciszek Hugon Szafraniec, Equivalent definitions

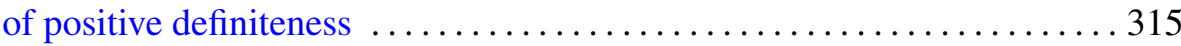

Costel Peligrad and S. Rubinstein, Maximal subalgebras of $C^{*}$-crossed

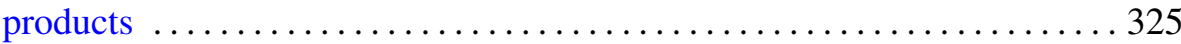

Derek W. Robinson and Sadayuki Yamamuro, Hereditary cones, order

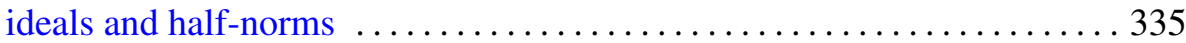

Derek W. Robinson and Sadayuki Yamamuro, The Jordan decomposition

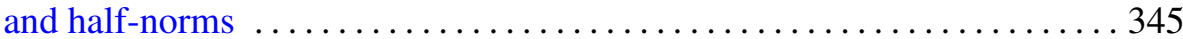

Richard Rochberg, Interpolation of Banach spaces and negatively curved

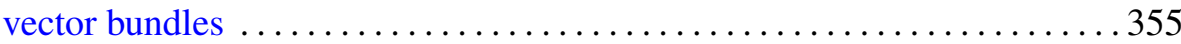

Dale Rolfsen, Rational surgery calculus: extension of Kirby's theorem 377

Walter Iaan Seaman, Helicoids of constant mean curvature and their Gauss maps

Diana Shelstad, Endoscopic groups and base change $\mathbf{C} / \mathbf{R}$

Jerrold Norman Siegel and Frank Williams, Numerical invariants of homotopies into spheres

Alladi Sitaram, Some remarks on measures on noncompact semisimple Lie groups

Teruhiko Soma, Atoroidal, irreducible 3-manifolds and 3-fold branched coverings of $S^{3}$

Jan de Vries, On the $G$-compactification of products

Hans Weber, Topological Boolean rings. Decomposition of finitely additive set functions 